\title{
Improved Technique for Gait Reconigition \& Performing Feature Extraction Using Videos of Different Forms
}

\author{
Ashutosh Batta ${ }^{1}$, Chirag Sharma ${ }^{2}$ \\ ${ }^{1}$ (M.C.A-Mtech (CSE) Lovely Professional University, Jalandhar India) \\ ${ }_{2}^{2}$ (Computer Science, Lovely Professional University, India)
}

\begin{abstract}
This paper describes the importance of GAIT Recognition where identification of human being from far distance without any sort of co-operation from his side is performed. Motive to develop automatic identification of human being using GAIT recognition method that to find an application which provide high security in places such as banking sector, military, parking slots. Work is performed on videos of different formats. In which extraction of frames from inputted video is done \& matched with trained database. Background subtraction is done to concentrate on particular object rather than complete frame \& irrelevant part is neglected. Feature extraction is done by taking an account of various parameters applied on the input and database to compare them. The significant part of this work is recognition in which we match the trained data with inputted data and then testing the result to check the accuracy of the applied technique.
\end{abstract}

Keywords: Gait recognition, Gait recognition approaches, multiple discriminant analysis, neural network, outermost contour, support vector machine.

\section{Introduction}

To detect and track the moving objects is widely used so that identification can be done in more efficient and effective way. For which the lots of techniques has already been proposed but every technique is having some advantages and disadvantages too. GAIT recognition is the one of the technique which is used to identify the individual while walking or running. It is the technique which provide the new way to biometric. Like in previous techniques which are available in to identify human being for example fingerprint, finger veins these techniques need interaction of human being which puts it at lower level as compare GAIT recognition which is non-invasive in nature. An early such attempts made by Lilli liu[1] to make the use of outermost contour then for recognition he has used different techniques like MDA,ENN,SVM,BPNN and also the collaborative techniques which shows the result maximum 97.67\% accuracy. Amit Kale [2] in his work firstly done on walking person then implemented the FED and HMM to lend the overall robustness to representation and recognition. M.Pushparani \& D.Sasikala made a gait recognition using the PCA and ICA then compared the results between them. Alese [3] used the model based approach, and then used the spatial and temporal metrics extracted from the model. ChewYean Yam [4] has done work on k-nearest neighbor classifier and the whole sequence of a gait cycle is described using the angle of thigh and lower leg rotation. Sanjeev Sharma [5] has done work on neural network after converting video into frames by which it achieved the accuracy of $96.32 \%$.

In fact, this paper is an extension of an earlier work done in the paper [1]. Now based on the extension to the base paper now going to make the contribution on following points, the main work of gait recognition is implemented in two parts which are normally known as testing and training part. In training part we give the input and implement the work at different steps and then in testing part we do the matching the database with the inputted video which was converted into the frames. So basically the gait recognition is divided into following parts to implement the work.

a. Firstly we carry out work on different types of videos having different extension; we are going to work on frames for which the inputted video or testing video will converted into the frames so that we can match them.

b. Secondly we will implement the concept of background subtraction so that unneeded part will be deleted and then we can concentrate on relevant part because if the threat is moving on a particular place but we need to concentrate only on object rather than complete frame.

c. In Third step, we are going to implement the concept of feature extraction which means on the basis of what different parameters we are going to match the database image with the man who is walking.

d. At last for better result and for effective and efficient result we will go core part of gait is implemented, recognition which is basically consists of two different parts:-

1. Matching the database with the inputted data.

2. Testing the result. 
At last we will check out the how much efficient CCR (correct classification rate).All of these steps will take place in two parts training and testing.

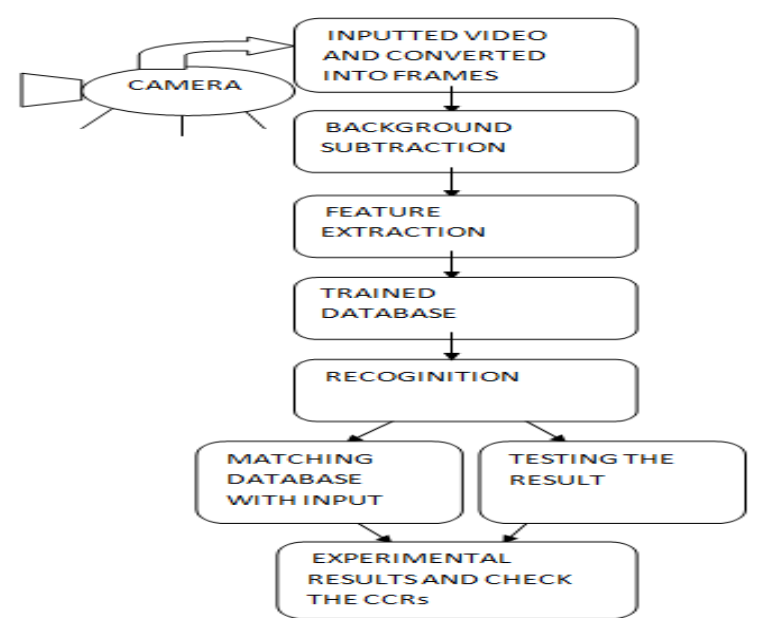

Figure 1: Development Steps of GAIT Recognition [11]

\section{Background Subtraction}

In this section we will implement the background subtraction concept in which we will just consider the relevant part and irrelevant part will be deleted for example we have complete frame where we have human being standing in the front of the building our main concentration is just on that human being not on building for which we use the concept of background subtraction

While implementing background subtraction we use the two different things Median filter and equation to do that task. The main reason to use the median filter is that it helps in to remove the noise and Median filter perform 2D median filtering [4]. In this block replaces the central value of an M-by-N neighborhood with its median value [5].

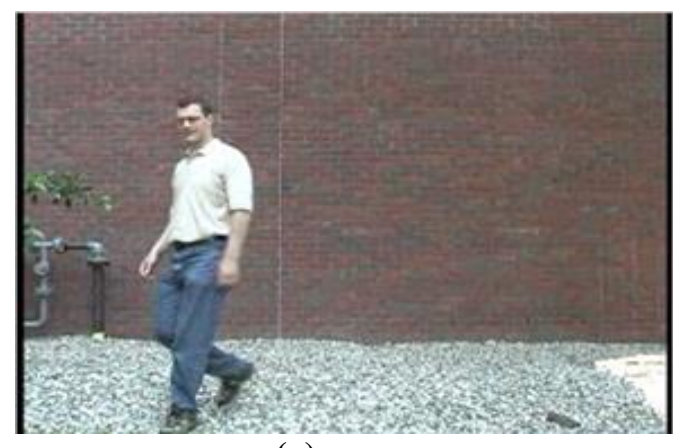

(a)

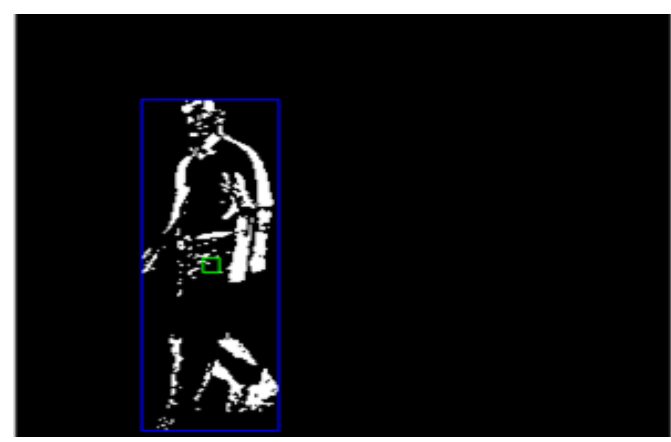

(b)

Figure 2: Working of Background Subtraction

\section{Feature Extraction}

In section we will introduce the concept of the feature extraction in which we will talk about the outermost contour and then Hanavan model and equations related to it.

In gait silhouette sequence, the gait depends upon the temporal changes of silhouette which is the only cue for identification[1], as my aim is to make the work like that which will reduce the cost and increase the effectiveness for which we use outermost contour.

In outermost contour we will work on the most right and left pixel on the contour belong to the outermost contour in each row of a normalized silhouette image [1]. While doing so to implement it we use the following equations, as firstly we need to compute the centroid $\left(x_{c}, y_{c}\right)$ of the outermost contour [1]. 


$$
\begin{gathered}
x_{c}=\frac{1}{n} \sum_{i=1}^{n} x_{i}, \\
y_{c}=\frac{1}{n} \sum_{i=1}^{n} y_{i}
\end{gathered}
$$

Where $\mathrm{n}$ is the number of pixels on the outermost contour, (xi, yi) is the coordinate of pixel on the outermost contour. Secondly, we compute the distance between each outermost contour pixel (xi; yi) and the centroid (xc, yc) row by row.

$$
d_{i}=\sqrt{\left(x_{i}-x_{c}\right)^{2}+\left(y_{i}-y_{c}\right)^{2}}
$$

As in outermost contour the number of pixels are definite in number so dimensionality cannot be reduced up to that extent for which we are going to use one more feature i.e. HANVAN'S MODEL ALGORITHM. The geometric human body model designed by Hanavan (1964).A total of 41 anthropometric parameters need to be measured in this model [9].
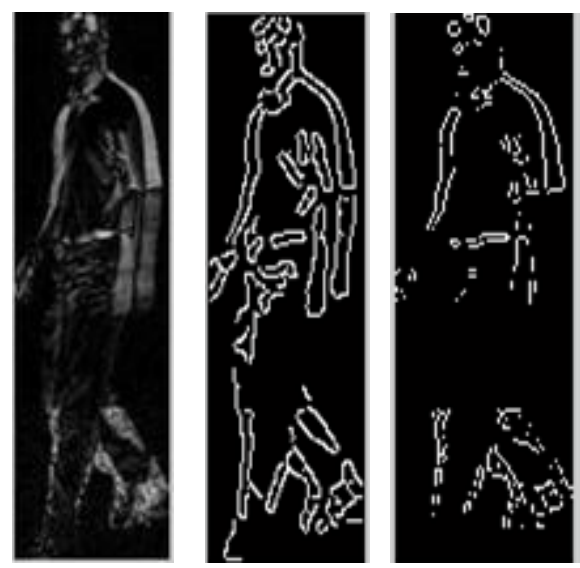

Figure 3: Working of Feature Extraction

\section{Matching \& Recognition}

The last step in implementing the gait process is to make the recognition which means to compare the results and before that we need to compare the inputted frame with database stored one to check whether they are same or not. For these steps we do the following work:-

For matching we use the simple and efficient method which is known as surf method to match the two different images or frames. Then to make our work more robust and effective we will implement the concept of SVM and NN as all these technique can be implemented only through the work done at previous steps.

\subsection{SVM method}

The Support Vector Machine (SVM) is a state-of-the-art classification method introduced in 1992 by Boser, Guyon, and Vapnik. The SVM classifier is widely used in bioinformatics (and other disciplines) due to its highly accurate, able to calculate and process the high-dimensional data such as gene expression and exibility in modeling diverse sources of data [10] .SVMs belong to the general category of kernel methods. A kernel method is an algorithm that depends on the data only through dot-products. When this is the case, the dot product can be replaced by a kernel function which computes a dot product in some possibly high dimensional feature space [10].

This has two advantages:

a) First, the ability to generate non-linear decision boundaries using methods designed for linear classifiers.

b) Second, the use of kernel functions allows the user to apply a classifier to data that have no obvious fixed-dimensional vector space representation [10]. 


\subsection{NN Method}

Neural Network is the concept which is mainly used in the image processing because of its result power and can be helpful for solving the multiple classification problems. It has lots of advantages like highly flexible, inductive and non-linear ability [1], because of which we are using it and by SVM+NN we can get the better results. While implementing the NN we will make the use of Delta rule, Error correction for the individual

\section{Experimental Results}

While implementing SVM at individual level with same steps at previous level we got the result $81.07 \%$ approx. For each testing sequence we firstly compute the gait feature which is known as feature extraction then we used the different techniques and the CCRs (Correct Classification Rate) are shown in the following table which show the different results of SVM , NN and at last purposed work done by us which is SVM+NN.

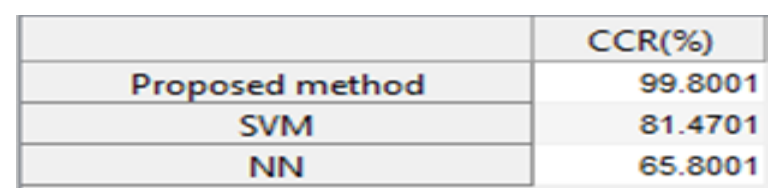

Figure 4: Achieved Results

The following cumulative graph shows the scores and shows how SVM+NN are more effective as compare to other methods.

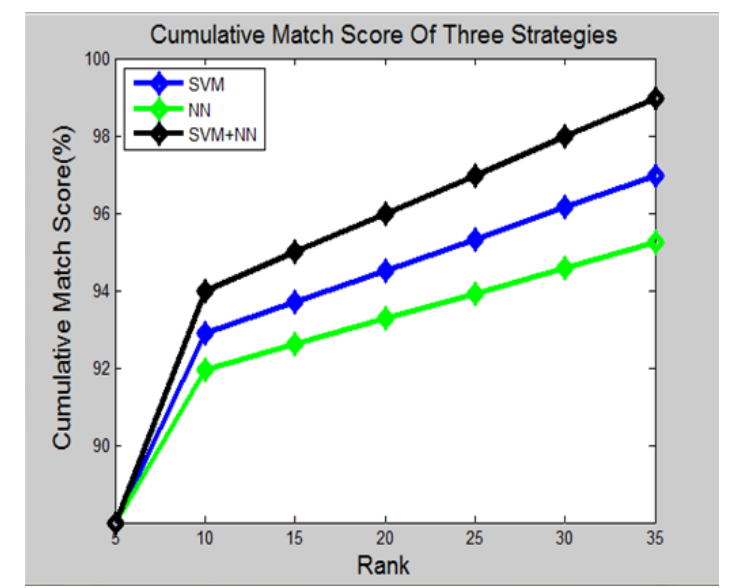

Figure 5: Shows SVM+NN better result as compare to other result

III. comparision

In This Section We Will Compare The Results of our work with work done by the Lilli liu [1] in which he has implemented the work like outermost contour and then PCA to gait feature and best results which he got from the MDA+ENN and lets us see the CCRs of our both methods

Table 1: Comparison of CCR's

\begin{tabular}{l|l}
\hline Recognition Methods & Best CCRs (\%) \\
\hline Lilli Liu & $\mathbf{9 7 . 6 7}$ \\
\hline Our Method & $\mathbf{9 9 . 8 0}$ \\
\hline
\end{tabular}

\section{Conclusion}

In this paper, we purpose a simple gait recognition technique method which is working on different sorts of videos as well as we have implemented median filter and then equation for background subtraction and then we have implemented outermost contour and Hanavan model at feature extraction level and then for matching we have used simple but effective method surf method and at last for recognition we have used the $\mathrm{MDA}+\mathrm{NN}$ which proves our results is more effective and efficient as compare to other purposed methods by different researchers and it show the accuracy of $99.80 \%$ achieved in this paper. This is method which makes gait recognition encouraging and research area for other who are thinking gait as future in the more recognition market. 


\section{References}

[1]. Liu, Yilong Yin, Wei Qin \& Ying Li: “Gait Recognition Based on Outermost Contour”, International Journal of Computational Intelligence Systems, 4:5, 1090-1099 (2011).

[2]. Amit Kale, Aravind Sundaresan, A. N. Rajagopalan, Naresh P. Cuntoor, Amit K. Roy-Chowdhury, Volker Kruger, and Rama Chellappa "Identification of Humans Using Gait"(IEEE TRANSACTIONS ON IMAGE PROCESSING, VOL. 13, NO. 9, SEPTEMBER 2004):

[3]. S John C. Russ.-- Russ, John C, The image processing handbook 5th ed. (2007).

[4]. ChewYean Yam, Mark S. Nixon, John N. Carter: "A Model-Based Approach Gait" Gait Recognition by Walking and Running: (January 2002,)

[5]. Sanjeev Sharma, Ritu Tiwari, Anupam shukla and Vikas Singh (2011): "Identification of People Using Gait Biometrics International Journal of Machine Learning and Computing", Vol. 1, No. 4, October 2011

[6]. Liang Wang, Tieniu Tan, Senior Member, IEEE, Huazhong Ning, and Weiming Hu "Silhouette Analysis-Based Gait Recognition for Human Identification"(IEEE TRANSACTIONS ON PATTERN ANALYSIS AND MACHINE INTELLIGENCE, VOL. 25, NO. 12, DECEMBER 2003)

[7]. M.Pushparani \& D.Sasikala Global Journal of Computer Science and Technology:” A Survey of Gait Recognition Approaches Using PCA \& ICA.” Volume 12 Issue 10 Version 1.0 May 2012 Type: Double Blind Peer Reviewed International Research Journal Publisher, Global Journals Inc. (USA) Online ISSN: 0975-4172 \& Print ISSN: 0975-4350: (May 2012)

[8]. "Motion and Feature Based Person Tracking in Surveillance Videos": 978-1-4244-7926-9/11/\$26.00 @2011 IEEE

[9]. http://www.kwon3d.com

[10]. Jyoti Sharma "An Efficient Technique of Image Noising and Denoising Using Neuro- Fuzzy and SVM (Support Vector Machine)A Survey" (IJCSIT) International Journal of Computer Science and Information Technologies.

[11]. Ashutosh Batta "Improving Technique for GAIT RECOGNITION \& Performing Feature Extraction Using Videos of Different Forms (IJCSIT) International Journal of Computer Science and Information Technologies 